\title{
The Electron Stopper Layer Effect on Long-Wavelength VCSEL with AsSb-Based DBR Temperature Distribution
}

\author{
Zahra Danesh Kaftrouddi ${ }^{a, *}$, Esfandiar Rajaei ${ }^{\text {b }}$ \\ ${ }^{a}$ Department of Engineering sciences, Faculty of Technology and Engineering East of Guilan, \\ University of Guilan, 44891-63157, Rudsar-Vajargah, Iran \\ ${ }^{b}$ Department of Physics, University of Guilan, 41335-1914, Rasht, Iran \\ *Corresponding Author's Email: Zahraadaneh@guilan.ac.ir
}

Received: May.4, 2016, Revised: Aug. 22, 2016, Accepted: Sep. 21, 2016, Available Online: Jan. 28,2017

DOI: 10.18869 /acadpub.ijop.11.1.25

\begin{abstract}
In this study, we have theoretically investigated the effect of electron stopper layer on internal temperature distribution of high performance vertical cavity surface-emitting laser emitting at $1305 \mathrm{~nm}$. Simulation software PICS3D, which selfconsistently combines the 3D simulation of carrier transport, self-heating, gain computation and wave-guiding, was used. Simulation results show that change the electron stopper layer properties affect the internal temperature distribution of the device. The temperature of the active region increases compared with the original device. Comparison of temperature distribution in devices with different electron stopper layer confirms that optimized structure operates at maximum temperature.
\end{abstract}

KEYWORDS: Vertical Cavity Surface Emitting Laser, Electron Stopper Layer, Temperature Distribution.

\section{INTRODUCTION}

Vertical cavity surface emitting laser (VCSEL) has a number of inherent advantages including low divergence of the circular beam, which is emitted from the top surface of the laser diode in order to efficiently couple into fiber for communication. One of the most unique characteristic operations of a VCSEL is that it operates in single longitudinal mode due to its natively short effective cavity length. Many researchers try to achieve high efficiency, high power, single transverse mode operation and insensitive temperature feature in VCSEL light-wave communication.

The design of long-wavelength InP-based VCSELs is very challenging compared with that of GaAs-based VCSELs due to the lack of an ideal DBR mirror, effective current and optical confinement method, and higher free carrier absorption in p-type materials. Instead of oxidation, InP-based VCSELs usually use tunnel junctions (TJs) to confine the current and optical mode [9], [10], [11]. The tunnel junction will reduce the total amount of $\mathrm{p}$ - type material, so the free carrier absorption will be minimized. An alternative method is to use proton implantation to isolate the materials outside of the current aperture and block the current flow [12], [13].

Despite their small $\Delta \mathrm{n}$ and thick DBR stacks, InP-based as-grown DBRs are still the most natural choice for long-wavelength VCSELs [14], especially for bottom n-type mirrors. However, p-type mirrors are usually less desirable because of their high free carrier absorption. A variety of dielectric DBR mirrors [15], GaAs-based DBR mirrors transferred by wafer fusion [16] and metalDBR hybrid mirrors [17] have been developed and applied in InPbased VCSELs. Dielectric DBR mirrors have very large $\Delta$ nvalues (> 1), so only a few pairs are needed to achieve more 
than $99.5 \%$ reflectivity. However, dielectric DBRs have very different thermal expansion coefficients compared with semiconductors, which could possibly cause some reliability issues. Fused GaAs DBR mirrors shares all of the advantages of $850 \mathrm{~nm}$ VCSELs but fusing two DBR mirrors onto an InP active region is rather complicated and costly. Nevertheless, monolithic mirror is desirable for InP based VCSELs. One solution to this problem is to utilize well-established InAlGaAs activeregion technology coupled with $\mathrm{AlGaAsSb}$ DBRs. This combination facilitates monolithicall-epitaxial InP-based devices spanning the entire $1300-1600 \mathrm{~nm}$ wavelength range.

Thermal design is very important for VCSELs. Compared with edge-emitting lasers, VCSELs usually have much smaller active regions and higher threshold current densities, which means that the temperatures of these active regions could be much higher. Additionally, thickbottomed DBRs are usually poor thermal conductors that trap heat in the active region. This problem is more serious in InP-based VCSELs because the thermal conductivity of the DBRs in these devices is usually 1 or 2 orders of magnitude lower than that in binary compound semiconductor materials [18].

VCSELs performance degradation at elevated temperature often requires the use of costly cooling devices. Much effort has been devoted to understand and overcome the high temperature failure of VCSELs used in telecommunication applications. Elevation of the external and/or internal laser temperature leads to reduced output power, higher threshold current, and lower slope efficiency.

For un-cooled operation, the unwanted part of carrier process, carrier leakage, affects device performance. Carrier leakage has been a big problem since the invention of VCSELs. The band offset of active region and waveguide layer form the barrier height. Carriers with higher energy than barrier can escape the QW. When temperature rises, there will be more carriers in the high-energy region and this increases carrier leakage. Electrons are more strongly affected than holes because electrons have much higher mobility and diffusion constant than holes.

The high temperature performance is indubitably related to the amount of current confined in the active region, which means that the high temperature performance in a laser device can be improved by raising the conduction band offset near the QW active region. Mostly, a thin high-band gap layer can be grown after the growth of the QW active region to prevent electrons from overflowing out of the QWs [19], [20], [21], and hence the carriers can be confined effectively to contribute to stimulated emission. This layer named electron stopper layer (ESL).

In prior study, we explained the effect of AlInAs electron stopper layer on the first above room temperature continuous wave lasing of $1305 \mathrm{~nm}$ InP-Based VCSEL implementing AsSb- based DBR performance [22]. In this paper, for the first time, the effects of AlInAs electron stopper layer properties on internal temperature distribution are investigated.

This paper is organized as follows. In Section II and III we introduce device structure and the simulator used in this study and discuss about the importance of calibration of material parameters. We present the simulation results in Section IV.

\section{VCSEL STRUCTURE}

We focus mainly on a single-mode, tunneljunction, bottom-emitting VCSEL structure. Figure 1 shows a schematic of the structure for a $1.3 \mu \mathrm{m}$ VCSEL. The investigated bottomemitting VCSEL was grown by solid-source molecular beam epitaxy in a single growth step. A double intracavity contacting scheme was employed to circumvent the high electrical and thermal resistances of the AsSbbased DBRs. The AlInGaAs active region contains five $1.0 \%$ compressively strained 7 $\mathrm{nm}$ quantum wells and six $0.6 \%$ tensilestrained 5-nm barriers. Claddings both sides of the active region are InP layers doped $5 \times 10^{17}$ $\mathrm{cm}^{-3}$ that facilitate current spreading and heat 
removal in the device. The total cavity thickness was $4-\lambda$. The device contains a $2 \times 10^{19} \mathrm{~cm}^{-3} \mathrm{n}^{++}-\mathrm{In} 0.52 \quad \mathrm{Al}_{0.29} \mathrm{Ga}_{0.19} \mathrm{As} / 2 \times 10^{20}$ $\mathrm{cm}^{-3} \mathrm{p}^{++}-\mathrm{In}_{0.52} \mathrm{Al}_{0.29} \mathrm{Ga}_{0.19} \mathrm{As}$ tunnel-junction layer.

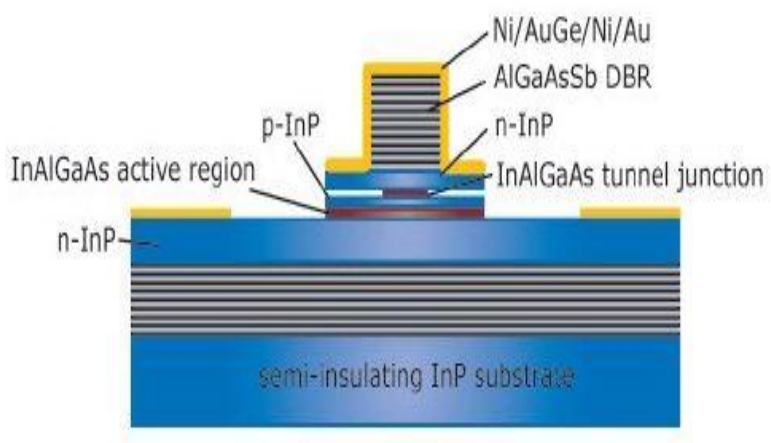

Fig. 1: Schematic of a $1.3 \mu \mathrm{m}$ VCSEL structure.

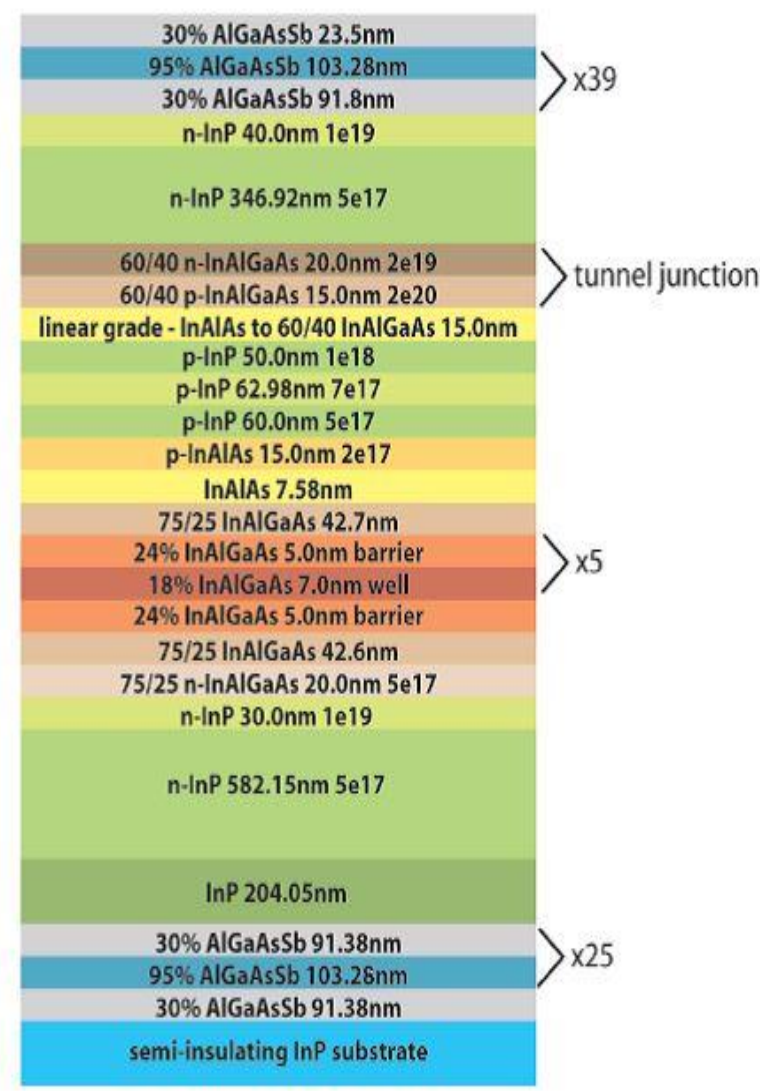

Fig. 2: Epitaxial structure grown for $1300 \mathrm{~nm}$ VCSELs.

Several p-type doping InP layers was used between the tunnel junction and the active region. The doping level was raised from $2 \times 10^{17} \mathrm{~cm}^{-3}$ to a grade of $1 \times 10^{18} \mathrm{~cm}^{-3}$ at the tunnel junction and $5 \times 10^{17} \mathrm{~cm}^{-3}$ near the active region. This scheme served to increase the carrier density of holes near the active region and thus improve the injection efficiency.

The epitaxial layer structure is shown in Fig. 2. The reader is noticed that a compound such as $60 / 40$ InAlGaAs refers to a quaternary composed of $60 \%$ of the lattice matched InAlAs ternary and $40 \%$ of the lattice matched InGaAs ternary. A compound such as $24 \%$ InAlGaAs simply means that the quaternary $\mathrm{Al}$ percentage is 24 .

The doping levels of semiconductor layers are given in Table1.

Table 1 Doping levels

\begin{tabular}{ll}
\hline \hline Material & Doping $\left(1 / \mathrm{cm}^{3}\right)$ \\
\hline \hline i- $\mathrm{Al}_{0.3} \mathrm{Ga}_{0.7} \mathrm{As}_{0.53} \mathrm{Sb}_{0.47}$ & - \\
i- $\mathrm{Al}_{0.95} \mathrm{Ga}_{0.05} \mathrm{As}_{0.51} \mathrm{Sb}_{0.49}$ & - \\
n-InP & $1 \times 10^{19}$ \\
n-InP & $5 \times 10^{17}$ \\
n- $\mathrm{In}_{0.52} \mathrm{Al}_{0.29} \mathrm{Ga}_{0.19} \mathrm{As}$ & $2 \times 10^{19}$ \\
p- $\mathrm{In}_{0.52} \mathrm{Al}_{0.29} \mathrm{Ga}_{0.19} \mathrm{As}$ & $2 \times 10^{20}$ \\
p-InP & $1 \times 10^{18}$ \\
p-InP & $7 \times 10^{17}$ \\
p-InP & $5 \times 10^{17}$ \\
p-InAlAs & $2 \times 10^{17}$ \\
i-InAlAs & - \\
i- $\mathrm{In}_{0.52} \mathrm{Al}_{0.36} \mathrm{Ga}_{0.12} \mathrm{As}$ & - \\
i- $\mathrm{In}_{0.43} \mathrm{Al}_{0.24} \mathrm{Ga}_{0.33} \mathrm{As}$ & - \\
i- $\mathrm{In}_{0.73} \mathrm{Al}_{0.21} \mathrm{Ga}_{0.06} \mathrm{As}$ & - \\
n-InP & $1 \times 10^{19}$ \\
n-InP & $5 \times 10^{17}$ \\
i-InP & - \\
i- $-\mathrm{Al}_{0.3} \mathrm{Ga}_{0.7} \mathrm{As}_{0.53} \mathrm{Sb}_{0.47}$ & - \\
i- $-\mathrm{AlAs}_{0.51} \mathrm{Sb}_{0.49}$ & - \\
i-InP & - \\
\hline \hline
\end{tabular}

\section{INTRODUCTION TO THE SIMULATOR}

Simulator for technology computer-aided design (TCAD) is already a fundamental part of silicon industry and has largely promoted the development of silicon-based devices [23].

As optoelectronic devices are gaining in importance in daily life appliance, simulators 
for their simulation analysis will also play a significant role in the near future. There are a variety of commercial tools available for device simulation with different types of physical models and numerical methods. The following simulation tools have been used extensively to model semiconductor laser diodes.

1- Minilase 2D laser simulator: This is a 2D laser simulator from the University of Illinois [24].

2- TCAD Sentaurus: This is a diverse 3D simulator from Synopsys [25].

3- DESSIS: This is a device simulator from ISE AG. It is multi dimension simulator and can perform 1D, 2D or 3D simulations [26].

4- TiberCAD: This is a 3D device simulator from the Opto and Nano Electronics group at the University of Roma [27].

The complex nature of VCSELs and the industry demand for their high reliability, excellent performance, and manufacture economics, require computer simulation for researchers to effectively analyze the different physical processes behind laser operation as well as the tangled interactions among them especially with shrinking sizes and new device concepts.

Simulation also helps us to evaluate the sensitivity of laser characteristics to various configurations and material properties, which is a key to identifying the crucial values of parameters for design considerations and performance optimization. However, VCSEL modeling once fell behind the experimental successes with the previous models focusing mainly on electronic transport processes and ignoring optical and thermal effects.

In the last decade, with the fast development of computer technology, fully coupled electroopto- thermal simulation study of a cylindrical symmetric VCSEL was reported, with results highly consistent with the experimental.
This simulation study is conducted by PICS3D (Photonic Integrated Circuit Simulator in 3D) which is a commercial laser simulator developed by Crosslight Software, Inc. [28]. PICS3D is considered as one mainstream simulator for optoelectronics in the market. By default PICS3D uses drift-diffusion theory to describe carrier transport in the bulk layers, together with a thermionic emission model as the boundary condition for heterojunctions [29]. A quantum well is treated as having two back-to-back heterojunctions and transport is mesh-point sequential: carriers from the left barrier must first drop into the well before they jump to the right barrier [30]. The current density including drift and diffusion components also takes thermal effects into account by an extra term describing the effect of temperature gradient on current distribution. The dependence of electric resistivity on carrier mobility is provided by the Hall measurement determining the values for maximum electron and hole mobility in GaAs. All the metal contacts are treated as ideal, which means no contact resistance or interference with optical waves.

In the vertical direction, the transfer matrix method (TMM) is used to evaluate the roundtrip gain and locate the position of the longitudinal modes. The optical modes couple to drift-diffusion model through carrierdependent refractive index. The temperature dependence of the refractive index is material dependent which has major influences on emission wavelength, DBR reflectivity as well as slope efficiency. The refractive index of each layer is updated according to local mesh temperature at each bias during a continuouswave simulation. The optical loss is governed by free-carrier absorption and Auger recombination. The free-carrier absorption is also referred as intraband absorption and is calculated by a linear combination of the contributions from electron and hole concentrations absorption. Another uniform background optical loss is also taken into account for non-active materials as a fitting parameter to compensate the uncertain loss mechanisms. 
Accurate thermal simulation results in correct temperature dependent material parameters among which the most important ones are carrier mobility, band gap energy, various recombination coefficients and refractive index. All of these temperature dependent parameters are evaluated and updated with local mesh temperatures at each bias point. The heat source can be separated into contributions from Joule heat, generation/recombination heat and Thomson/Peltier heat. Heat flux is governed by material thermal conductivity defined as function of both material composition and local temperature.

The calculation of the quantum wells gaincurrent relationship requires among the most sophisticated models in the simulation and there are different levels of approximations to include in the calculations. In this work, the default quantum model was applied which assumes a single, symmetric, flat-band and step-wise potential profile. Quantum wells in a MQW region are assumed to be isolated from each other and wave functions do not overlap. The quantum well levels are calculated at every bias point during an actual simulation because the band gap of the active region is a function of carrier density. As the bias increases, the higher carrier density in the quantum well reduce the band gap and changes the quantum well depth. A full computation of subbands using k.p theory is performed taking the strain induced nonparabolic valence band splitting and mixing into account. Carrier densities and interband optical transitions are obtained using numerical integral over the non-parabolic subbands, resulting in longer computation time.

The accuracy of simulations depends on the correct choice of the physical models and material parameters. However, apart from the fact that accurate data for material parameters of semiconductor compounds is not always available, the overall complexity of diode lasers, combining semiconductor electronics and photonics, makes this a challenging task. Moreover, the actual composition and thickness of epitaxial grown layers, especially in the active region, may deviate from the anticipated values due to fluctuations in the growth parameters. This also contributes to the uncertainty in the simulation results. Thus, careful adjustments of material parameters are required to find agreement with measurements. In our previous study, we explained how to extract these material parameters from experimental results [31].

Table 2 Material parameter of the binary semiconductor used in this study.

\begin{tabular}{lllllll}
\hline \hline Parameter & GaAs & InAs & AlAs & AlSb & GaSb & InP \\
\hline \hline $\begin{array}{l}\text { Electron } \\
\text { eff. Mass }\end{array}$ & 0.0665 & 0.027 & 0.15 & 0.14 & 0.039 & 0.064 \\
$\begin{array}{l}\text { Luttinger } \\
\text { parameter1 }\end{array}$ & 6.85 & 19.67 & 3.76 & 5.18 & 13.4 & 6.35 \\
$\begin{array}{l}\text { Luttinger } \\
\text { parameter2 }\end{array}$ & 2.1 & 8.37 & 0.82 & 1.19 & 4.7 & 2.08 \\
$\begin{array}{l}\text { Luttinger } \\
\text { parameter3 }\end{array}$ & 2.9 & 9.29 & 1.42 & 1.97 & 6.0 & 2.76 \\
$\begin{array}{l}\text { Lattice } \\
\text { constants }\end{array}$ & 5.65325 & 6.058 & 5.6611 & 6.163 & 6.096 & 5.869 \\
$\begin{array}{l}\text { Elastic } \\
\text { constant1 }\end{array}$ & 11.81 & 8.329 & 12.5 & 8.769 & 8.842 & 10.22 \\
$\begin{array}{l}\text { Elastic } \\
\text { constant2 }\end{array}$ & 5.32 & 4.526 & 5.43 & 4.341 & 4.026 & 5.79 \\
\hline \hline
\end{tabular}

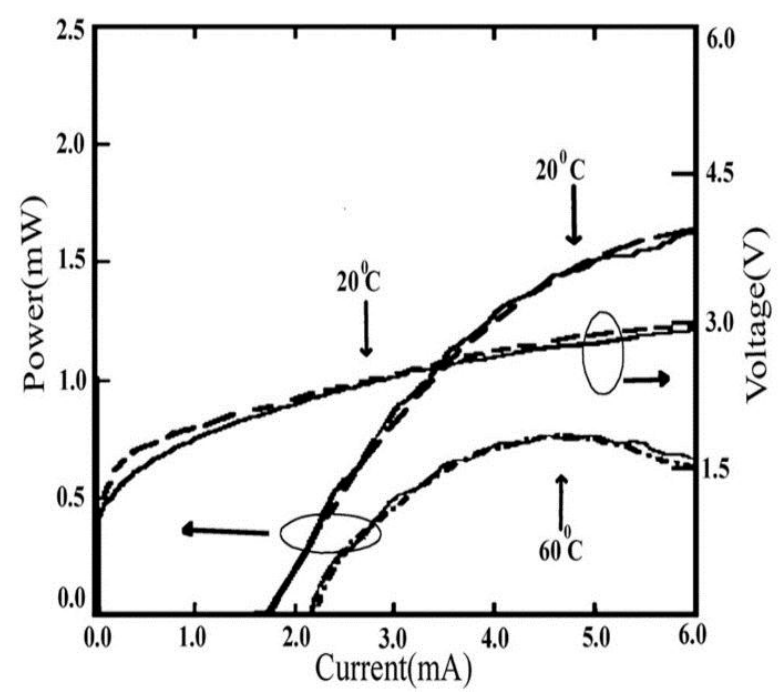

Fig. 3 Measured (solid line) and simulated (dashed line) L-I and I-V characteristics in CW operation at different temperatures.

In this study, the relevant parameters for the $\mathrm{In}_{1-\mathrm{x}-\mathrm{y}} \mathrm{Ga}_{\mathrm{x}} \mathrm{Al}_{\mathrm{y}} \mathrm{As}$ material system are linearly interpolated between the parameters of the relevant binary semiconductor. The material 
parameters of the binary semiconductor used in the simulation are listed in Table 2.

These parameter lead to good agreement between simulations and measurements at 20 ${ }^{\circ} \mathrm{C}$ and $60{ }^{\circ} \mathrm{C}$ stage temperatures as shown in Fig. 3.

In the following of this Section, the summarized of the laser model will be explained.

\section{A. Electrical Model}

The electrical behavior of an InP-based semiconductor device is described with finiteelement drift-diffusion model in this package:

$$
\begin{aligned}
& -\nabla \cdot\left(\frac{\varepsilon_{0} \varepsilon_{d c}}{q} \nabla V\right)=-n+p+N_{D}\left(1-f_{D}\right) \\
& -N_{A}+\sum_{j} N_{t}\left(\delta_{j}-f_{t j}\right) . \\
& \nabla \cdot \mathbf{J}_{\mathbf{n}}-\sum_{j} R_{n}^{t j}-R_{s p}-R_{s t}-R_{A u g}+G_{o p t}(t) \\
& =\frac{\partial n}{\partial t}+N_{D} \frac{\partial f_{D}}{\partial t} . \\
& \nabla \cdot \mathbf{J}_{\mathbf{p}}+\sum_{j} R_{p}^{t j}+R_{s p}+R_{s t}+R_{A u g}-G_{o p t}(t) \\
& =-\frac{\partial p}{\partial t}+N_{A} \frac{\partial f_{A}}{\partial t} .
\end{aligned}
$$

The model is primarily governed by three equations. One is Poisson's equation, shown in Eq. (1). The others are current continuity equation for electrons and hole shown in Eq.(2) and (3), respectively. In Eq.(1), $\varepsilon_{0}$ and $\varepsilon_{d c}$ are dielectric constants of vacuum and relative dielectric constant, respectively. Also $n$ and $p$ are electron and hole concentrations. $N_{D}$ and $N_{A}$ are doping densities of shallow donors and shallow acceptors. $N_{t j}$ is density of $\mathrm{j}_{\text {th }}$ deep trap, $f_{D}$ and $f_{A}$ are occupancies of donor and acceptor levels and $f_{t j}$ is occupancy of the $\mathrm{j}_{\text {th }}$ deep trap level. In Eq. (2) and (3), $G_{\text {opt }}$ is the photon generation rate per unit volume. Also $R_{s t}, R_{s p}$ and $R_{A u g}$ are stimulated, spontaneous and Auger recombination emission rate, respectively.

\section{B. Optical Model}

The software solves the scalar Helmholtz equation: $\frac{\partial^{2} \varphi}{\partial x^{2}}+\frac{\partial^{2} \varphi}{\partial y^{2}}+\left(k^{2}-\beta^{2}\right) \varphi=0$

Where $\varphi(x, y)$ represents any transverse component of the optical field, $k$ is the absolute value of the wave vector and $\beta$ is the longitudinal propagation constant. The lateral optical components are given by Bessel functions which are adjusted to measured VCSELs near fields.

\section{Heat Generation and Dissipation}

For the thermal simulation, software solves the steady-state heat flux equation.

$$
\rho_{L} C_{L} \frac{\partial T}{\partial t}=-\nabla \cdot \mathbf{J}_{\text {Heat }}+H_{\text {heat }} .
$$

Where $H_{\text {heat }}$ is the heat source and $\rho_{L}$ is material density. The heat source can be separated in to contributions from Joule heat, generation / recombination heat, absorption heat and Thomson heat.

\section{DISCUSSION OF THE RESULTS OF SiMULATIONS}

For a proper laser operation, not only at higher outer temperatures but also at room temperature, carefully designed thermal properties of the device are of the great importance, since the internal temperatures inside of the laser can be much higher than the ambience temperature. If semiconductor lasers operating at high outer temperatures, e.g. $85^{\circ} \mathrm{C}$ or higher, should be realized, thermal phenomena inside of the device become one of the crucial laser design aspects. It is of great importance to have a deeper understanding of the thermal processes in a laser, to know the heat generation and heat dissipation mechanisms. Because of the energy conservation, the part of the supplied electrical energy, which is not converted to optical energy inside of a laser, will generate heat in the device, strongly affecting device performance. The two major tasks of VCSEL thermal designing are to reduce the heat generation inside of the device and to carry off the heat, which have been generated, efficiently. Of course, it is better not to have 
the heat at all or to have only a small amount of it, than to generate much heat and to try to carry it off. In order to reduce heat generation, corresponding mechanisms should be studied. In a practical device a significant amount of the heat will be commonly still present, so heat flux phenomena should be considered as well.

To understand and effectively reduce the heat generation, corresponding phenomena should be considered. In a semiconductor laser heat is generated by different mechanisms. The most relevant are the Joule heat, the electron-hole recombination heat, the Thomson heat and the heat from optical absorption. The Joule heat (which is directly proportional to the electrical resistance of the material) is generated by the carrier flow through a semiconductor and the corresponding scattering by phonons [32]. The recombination heat is generated by nonradiative recombination processes of the electron-hole pairs. These processes include defect and impurity recombination, recombination on surfaces and interfaces and Auger recombination. The average recombination heat is proportional to the difference between the quasi-Fermi levels. The Thomson heat results from the differences in the thermoelectric power, which is the measure for the increase in average carrier excess energy with increasing temperature. A dramatic example is the interface between different semiconductors, like in a DBR mirror. By moving through an interface from a material with the larger band gap to a material with the smaller band gap, electrons exhibit excess kinetic energy (hot electrons), which can dissipate to the lattice. In the opposite case electrons needs to receive some energy from the lattice. Thus the Thomson heat can be both positive and negative. The optical absorption heat arises from the absorption of photons in the semiconductor material. Photons can be directly absorbed by the crystal lattice, but at the typical photon energies considered here the free carrier absorption is dominating. The absorbed energy is then quickly dissipated to the lattice due to very short intraband scattering times. The main conclusion is that the location of heat sources is of major importance for the active region temperature increase.

In our previous work, the electron stopper layer effects on the device performance were investigated and the optimum layer properties were obtained [22]. Here, we studied the effect of the ESL on the device temperature distribution. We focused on the active region temperature.

The 2- dimensional temperature distribution of devices with different electron stopper layer thicknesses are plotted in Fig.s 4 to 6 at maximum power when the stage temperature is $60^{\circ} \mathrm{C}$.

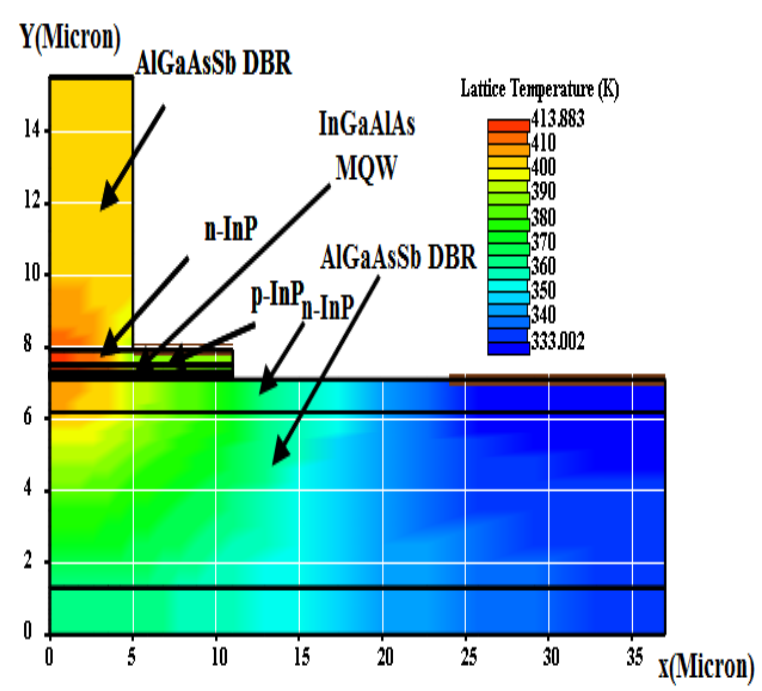

Fig. 4: Two- dimensional temperature distribution of laser with $10 \mathrm{~nm}$ electron stopper layer thickness at maximum power for $60^{\circ} \mathrm{C}$ stage temperature.

From these figures, we can clearly deduce that, the temperature of central section of VCSEL is higher than other section. This means that a large amount of heat generated in this region.

Since optical wave intensity confined in center of VCSEL and recombination processes (radiative and non-radiative) occur in this region.

Generated heat from the center of VCSEL flow laterally and vertically downward toward the InP substrate through bottom epitaxial DBR and also toward the top DBR. Since the 
top DBR is close to highly p-doped layer (Joul Heating Source), the top DBR is hotter than bottom DBR. It is noted that the DBRs in intracavity contacted VCSELs have no Joule heat generation because no current flow occurs through both top/bottom DBR mirrors.

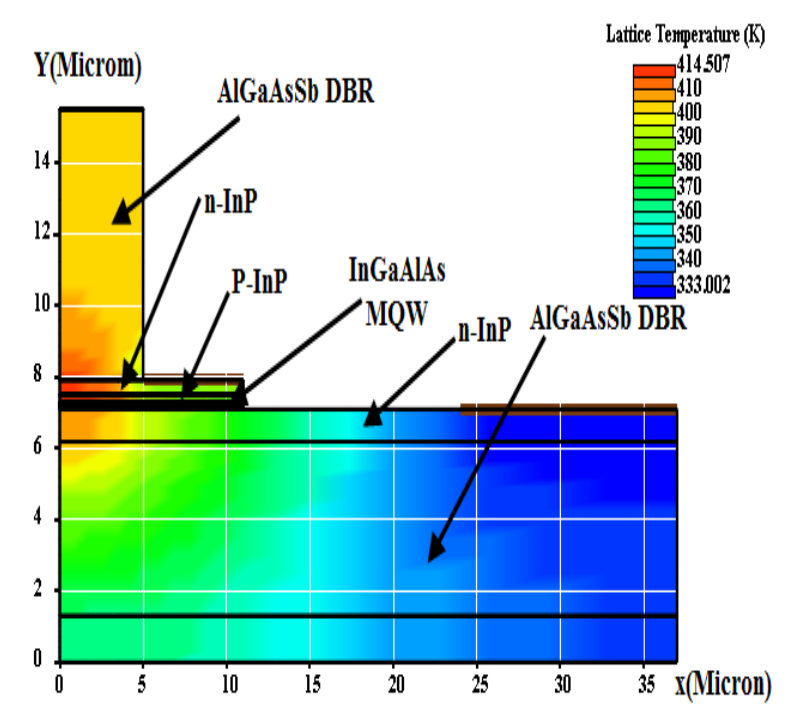

Fig. 5: Two- dimensional temperature distribution of laser with $15 \mathrm{~nm}$ electron stopper layer thickness at maximum power for $60{ }^{\circ} \mathrm{C}$ stage temperature.

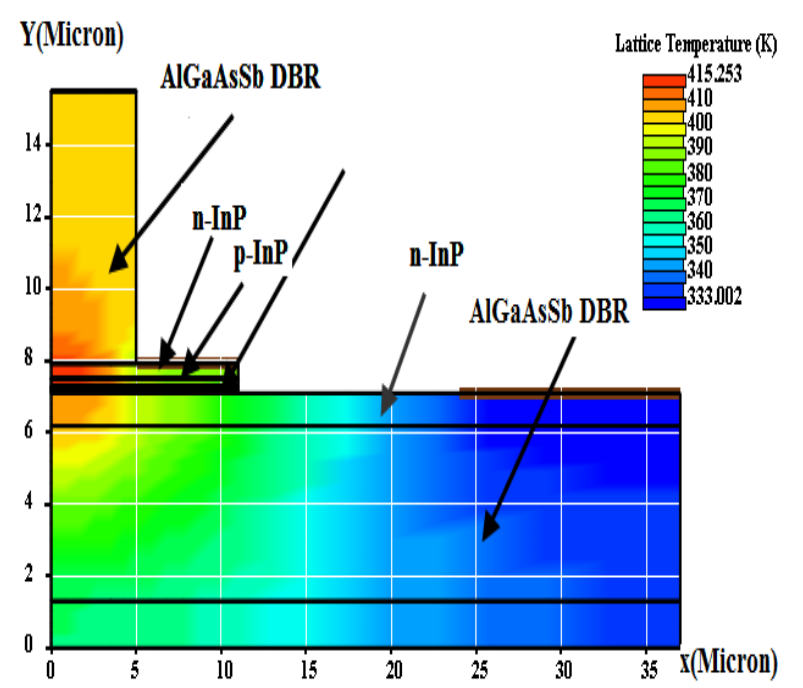

Fig. 6: Two- dimensional temperature distribution of laser with $20 \mathrm{~nm}$ electron stopper layer thickness at maximum power for $60^{\circ} \mathrm{C}$ stage temperature.

As seen in Fig. 7, the top DBR temperature is constant, but the bottom DBR temperature increases linearly in vertical direction. It also shows that, maximum temperature is located in active region.
The heat sources are mainly distributed in the active region by nonradiative recombination and absorption of spontaneous emission of light, negligible Joule heating [33].

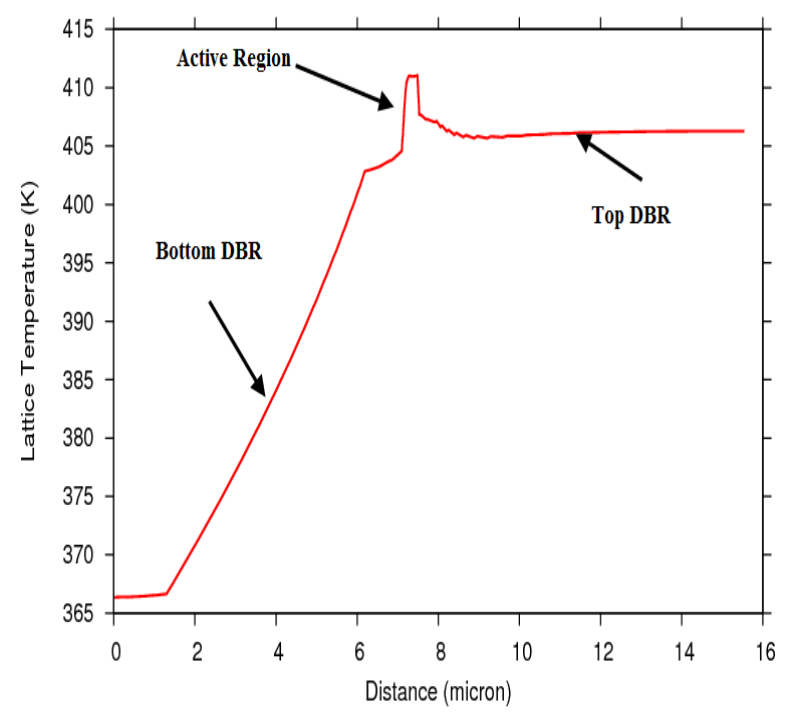

Fig. 7: Laser temperature vs distance at maximum power for $60{ }^{\circ} \mathrm{C}$ stage temperature with $10 \mathrm{~nm}$ electron stopper layer thickness.

\section{Y(Micron)}

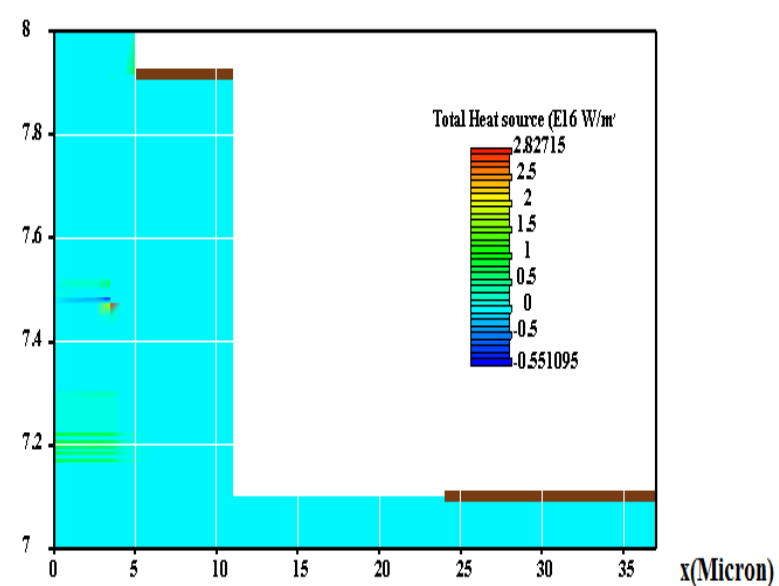

Fig. 8: Two- dimensional heat power distribution of laser with $10 \mathrm{~nm}$ electron stopper layer thickness at maximum power for $60^{\circ} \mathrm{C}$ stage temperature.

These figures show that the active region temperature increases about $13^{\circ} \mathrm{C}$ compared with original design [22]. The maximum internal temperature, which is caused by the heat generated inside the device, is raised up to $413.883 \mathrm{~K}, 414.507 \mathrm{~K}$ and $415.253 \mathrm{~K}$ in the active region for devices with $10 \mathrm{~nm}, 15 \mathrm{~nm}$ and $20 \mathrm{~nm}$ ESL thicknesses, respectively. 
With increasing the electron stopper layer thickness from $10 \mathrm{~nm}$ to $20 \mathrm{~nm}$ the active temperature rises from $140.883^{\circ} \mathrm{C}$ to $142.253^{\circ} \mathrm{C}$. The reason of such temperature climbing can be understood by considering heat power distribution in the devices with different electron stopper layer thicknesses.

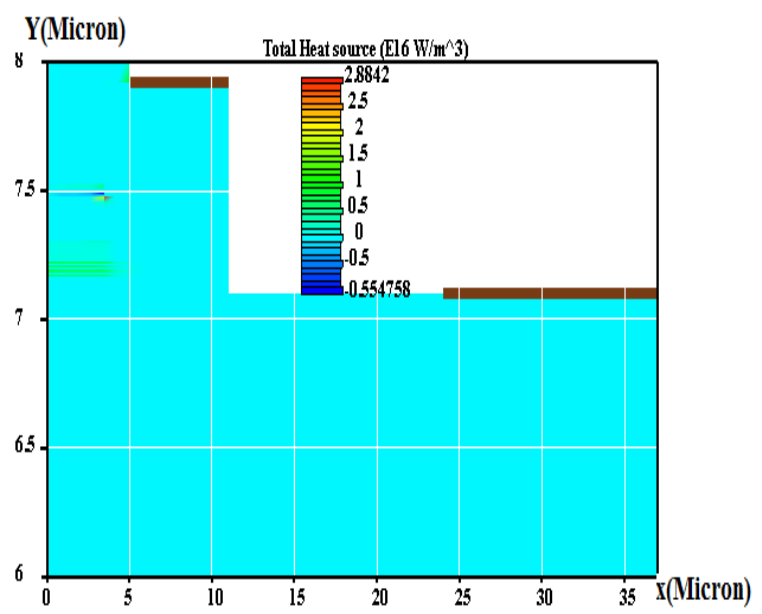

Fig. 9: Two- dimensional heat power distribution of laser with $15 \mathrm{~nm}$ electron stopper layer thickness at maximum power for $60^{\circ} \mathrm{C}$ stage temperature.

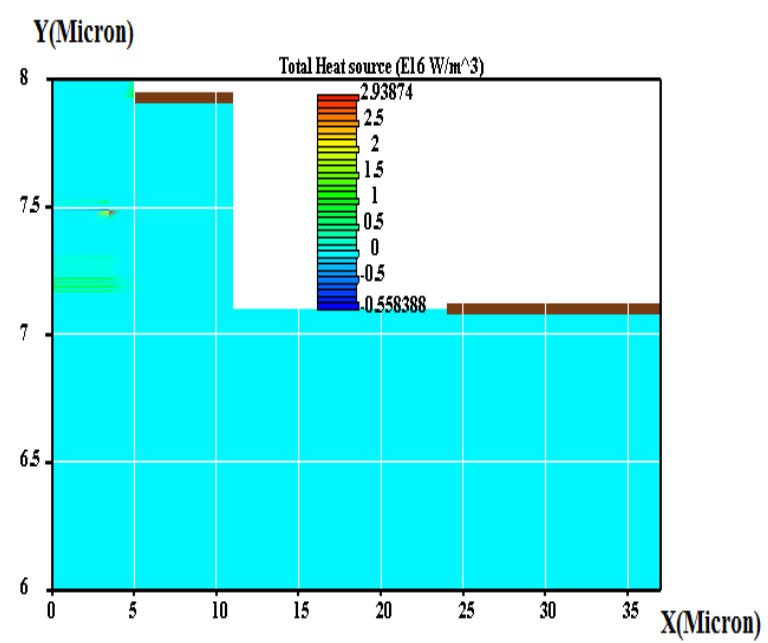

Fig. 10: Two- dimensional heat power distribution of laser with $20 \mathrm{~nm}$ electron stopper layer thickness at maximum power for $60{ }^{\circ} \mathrm{C}$ stage temperature.

The heat power is define as the power provided by the current injection and optical absorption, from which power converted into the stimulated emission power is subtracted.

Figures 8 to 10 compare total generated heat power density in the three mentioned devices.
As shown in these figures, the generated heat power in the active region with $20 \mathrm{~nm}$ ESL is slightly higher than other devices. This means that by increasing the AlInAs thickness heat removal from the active region occurs less, it is acceptable because the thermal conductivity decreases by thickness increasing. Thus the active region temperature increases.

At next step, the location of ESL layer was changed and temperature distribution for devices with different ESL offsets was obtained.

Figures 11 and 12 show the temperature distribution for VCSELs with $5 \mathrm{~nm}$ and $10 \mathrm{~nm}$ ESL offset, respectively.

From these figures, we can clearly deduce two things. First the active region temperature decreases in comparison with zero offset (correspond to placed above the last barrier); Second the active temperature decrease by moving the ESL layer away from last barrier.

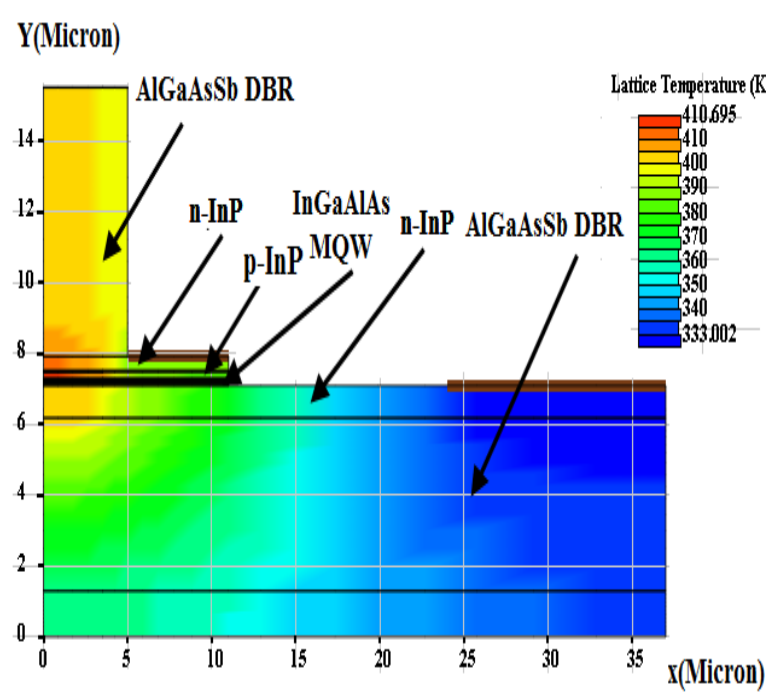

Fig. 11: Two- dimensional temperature distribution of laser with $5 \mathrm{~nm}$ electron stopper layer offset at maximum power for $60^{\circ} \mathrm{C}$ stage temperature.

The reason for such changes in the temperature of the active region is understandable considering the distribution of heat sources.

Total heat sources for two structures are presented in Fig. 13 and 14. As seen in these 
figures, the heat power density decreases as ESL offset increases. As a consequence the active region temperature decreases.

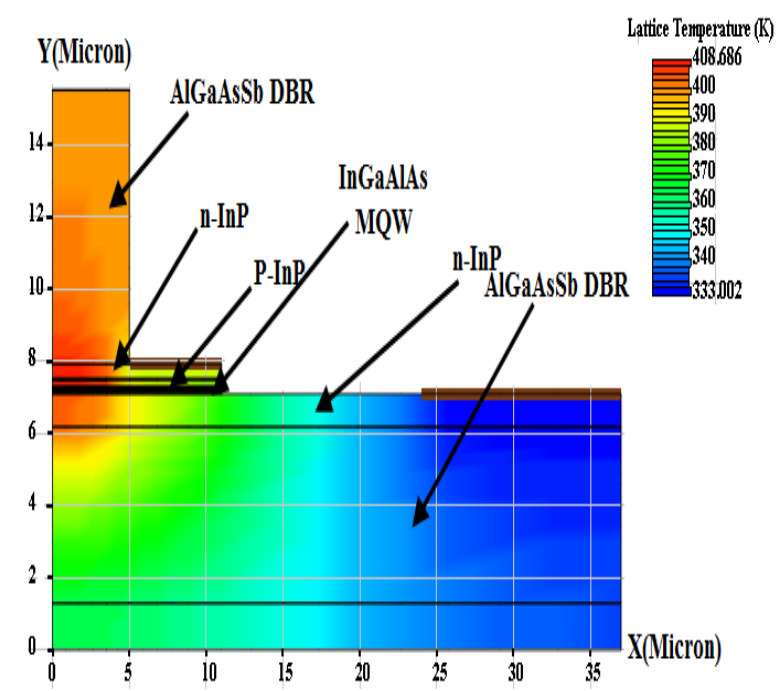

Fig. 12: Two- dimensional temperature distribution of laser with $10 \mathrm{~nm}$ electron stopper layer offset at maximum power for $60{ }^{\circ} \mathrm{C}$ stage temperature.

\section{Y(Micron)}

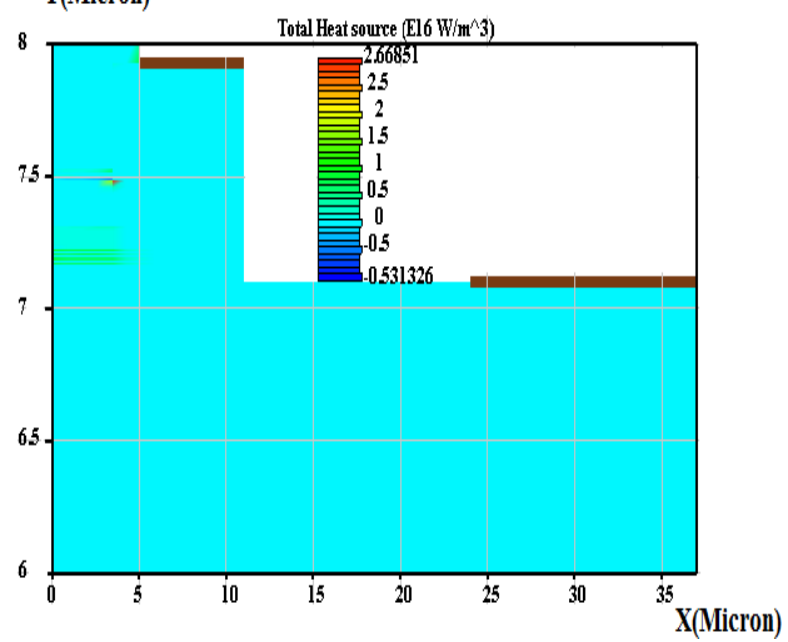

Fig. 13: Two- dimensional heat power distribution of laser with $5 \mathrm{~nm}$ electron stopper layer offset at maximum power for $60{ }^{\circ} \mathrm{C}$ stage temperature.

Other property of ESL layer is layer doping level. For optimum thickness $(20 \mathrm{~nm})$ and offset $(0 \mathrm{~nm})$, the layer doping level was changed and the temperature distribution was obtained. Figures 15 and 13 show the temperature distribution of devices with $5 \times 10^{23} \mathrm{~cm}^{-3}$ and $7 \times 10^{23} \mathrm{~cm}^{-3} \mathrm{p}$ - doping level, respectively.

The maximum temperature does not vary significantly with increasing the $\mathrm{p}$ - doping level. But the active region temperature of optimized structure (Fig. 16) has fallen slightly in comparison with the three first devices which mentioned above.

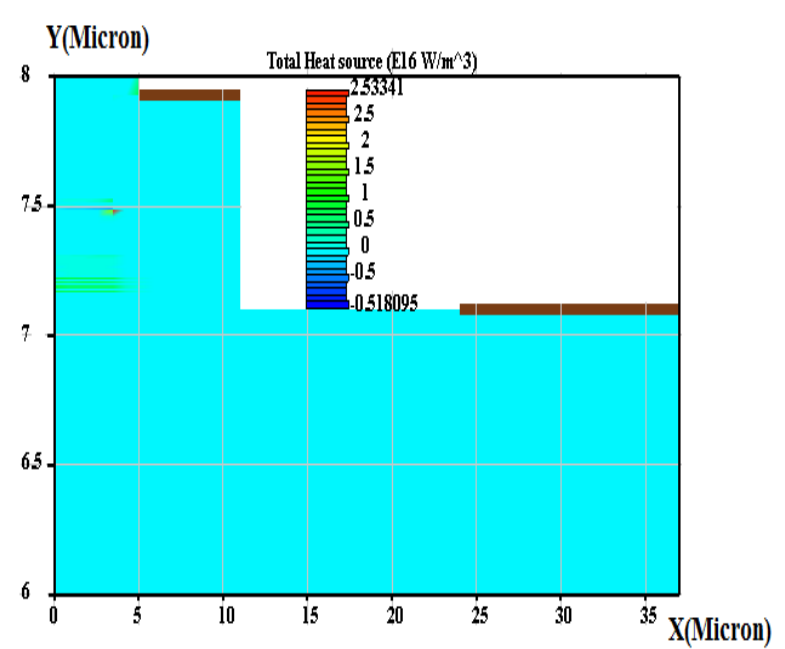

Fig. 14: Two- dimensional heat power distribution of laser with $10 \mathrm{~nm}$ electron stopper layer offset at maximum power for $60^{\circ} \mathrm{C}$ stage temperature.

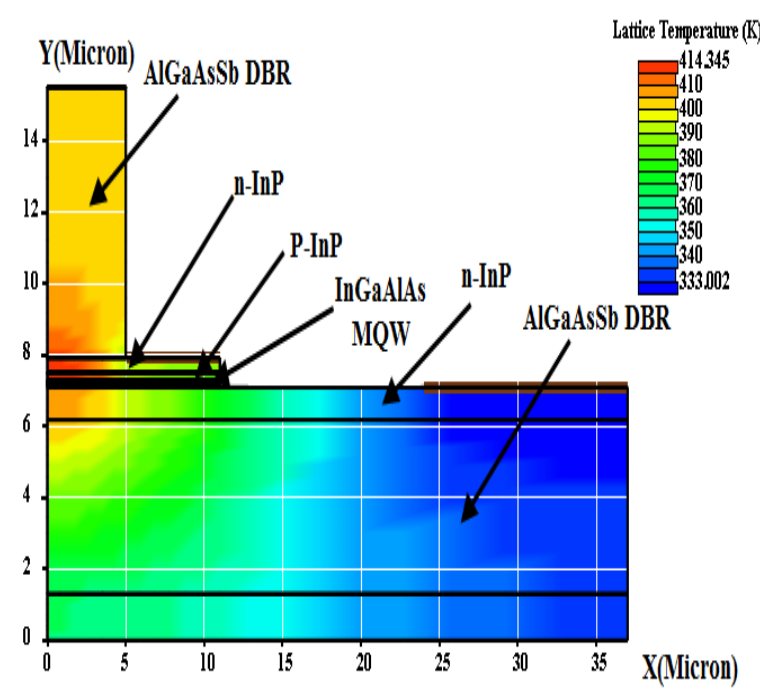

Fig. 15: Two- dimensional temperature distribution of laser with $20 \mathrm{~nm}$ electron stopper layer thickness and with $5 \times 10^{23} \mathrm{~cm}^{-3} \mathrm{p}$ - doping level at maximum power for $60{ }^{\circ} \mathrm{C}$ stage temperature.

The temperature distribution of optimum device [22] is plotted in Fig. 17.

By comparing all structures, it is obvious that the optimized device can operate at maximum temperature, so it has better thermal stability. 


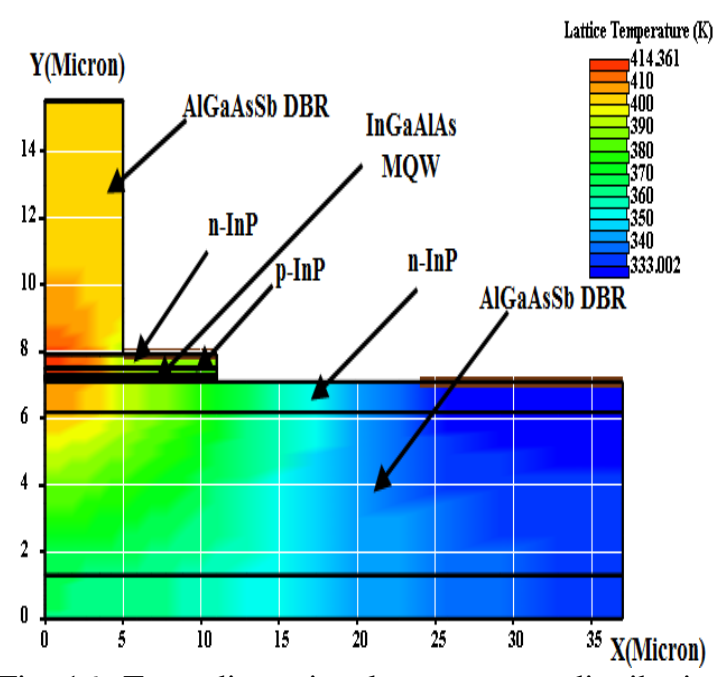

Fig. 16: Two- dimensional temperature distribution of laser with $20 \mathrm{~nm}$ electron stopper layer thickness and with $7 \times 10^{23} \mathrm{~cm}^{-3} \mathrm{p}$ - doping level at maximum power for $60{ }^{\circ} \mathrm{C}$ stage temperature.

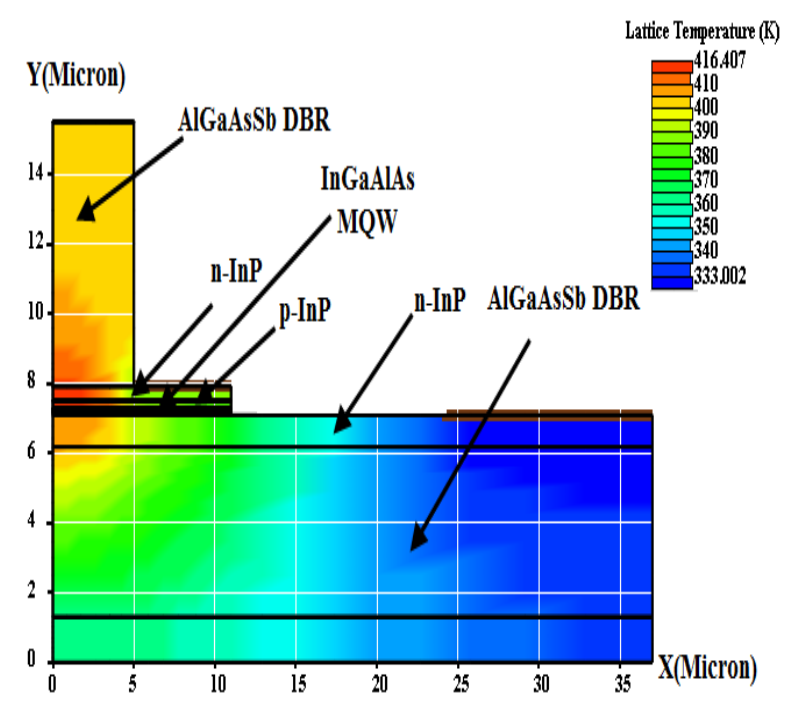

Fig. 17: Two- dimensional temperature distribution of optimized device at maximum power for $60{ }^{\circ} \mathrm{C}$ stage temperature.

\section{CONCLUSION}

In conclusion, we demonstrated that the combination of laser measurements with advanced simulations has enabled a detailed study of laser performance. Key material parameters of the model were calibrated using experimental characteristics. The resulting agreement between measurements and simulations validates the laser model serving as a starting point for a detailed study of the internal temperature distribution in devices with different ESL. We have demonstrated that the active region temperature changed with specification changes of ESL.

It is shown that the optimized structure can operate at maximum temperature.

\section{ACKNOWLEDGMENT}

We express our sincere appreciation to the Managers of Cross Light Inc. for providing us with the advanced three-dimensional PICS3D simulation program (version 2008.12) and their kind support.

\section{REFERENCES}

[1] F. Koyama, "Recent advances of VCSEL photonics,"J. Light wave. Technol. Vol. 24, No. 12, pp. 4502-4513, 2006.

[2] M. Ortsiefer, R. Shau, G. Bohm, F. Kohler, and M. C. Amann, "Low-threshold indexguided $1.5 \mu \mathrm{m}$ long-wavelength verticalcavity surface-emitting laser with high efficiency," Appl. Phys. Lett. Vol. 76, No. 16, pp. 2179-2181, 2000.

[3] W. Yuen, G. Li, R. Nabiev, J. Boucart, P. Kner, R. Stone, D. Zhang, M. Beaudoin, T. Zheng, C. He, M. Jensen, D. Worland, and C. Chang-Hasnain, "High-performance $1.6 \mu \mathrm{m}$ single-epitaxy top-emitting VCSEL," Electron. Lett. Vol. 36, No. 13, pp. 11211123, 2000.

[4] N. Nishiyama, C. Caneau, B. Hall, G. Guryanov, M. H. Hu, X. S. Liu, M.-J. Li, R. Bhat, and C. E. Zah, "Long-wavelength vertical-cavity surface-emitting lasers on InP with lattice matched AlGaInAs-InP DBR grown by MOCVD," IEEE J. Sel. Topics Quantum Electron. Vol. 11, No. 5, pp. 990998, 2005.

[5] M.C. Amann and W. Hofmann, "InP-based long-wavelength VCSELs and VCSEL arrays," IEEE J. Sel. Topics Quantum Electron, Vol. 15, No. 3, pp. 861-868, 2009.

[6] A. Mereuta, G. Suruceanu, A. Caliman, V. Iacovlev, A. Sirbu, and E. Kapon, "10-Gb/s and $10-\mathrm{km}$ error-free transmission up to $100 \mathrm{C}$ with $1.3 \mu \mathrm{m}$ wavelength wafer-fused VCSELs," Opt. Express, Vol. 17, No. 15, pp. 12 981-12986, 2009.

[7] W. Hofmann, M. Muller, A. Nadtochiy, C. Meltzer, A. Mutig, G. Bohm, J. Rosskopf, D. Bimberg, M.-C. Amann, and C. Chang- 
Hasnain, "22-Gb/s long wavelength VCSELs," Opt. Express, Vol. 17, No. 20, pp. 17 547-17 554, 2009.

[8] M. Muller, W. Hofmann, G. Bohm, and M.C. Amann, "Short-cavity long-wavelength VCSELs with modulation bandwidths in excess of $15 \mathrm{GHz}$," IEEE Photon. Technol. Lett. Vol. 21, No. 21, pp. 1615-1617, 2009.

[9] E. Kapon and A. Sirbu, "Long-wavelength VCSELs: Power-efficient answer," Nature Photonics, Vol. 3, pp. 27-29, 2009.

[10] N. Nishiyama, C. Caneau, G. Guryanov, X. S. Liu, M. Hu, and C.E. Zah, "High efficiency long -wavelength VCSEL on InP grown by MOCVD," Electron. Lett. Vol. 39, No. 5, pp. 437-439, 2003.

[11]M. Ortsiefer, S. Baydar, K. Windhorn, G. Bohm, J. Rosskopf, R. Shau, E. Ronneberg, W. Hofmann, and M. C. Amann, " $2.5-\mathrm{mW}$ single-mode operation of $1.55-\mu \mathrm{m}$ buried tunnel junction VCSELs," IEEE Photon. Technol. Lett. Vol. 17, No. 8, pp. 1596-1598, 2005.

[12] J. Cheng, C.L. Shieh, X. Huang, G. Liu, M.V.R. Murty, C.C. Lin, and D. X. Xu, "Efficient CW lasing and high-speed Modulation of $1.3-\mu \mathrm{m}$ AlGaInAs VCSELs with good high temperature lasing performance," IEEE Photon. Technol. Lett. Vol. 17, No. 1, pp. 7-9, 2005.

[13]C. J. Chang-Hasnain, J.P. Harbison, G. Hasnain, A.C.V. Lehmen, L.T. Florez, and N.G. Stoffel, "Dynamic, polarization, and transverse mode characteristics of vertical cavity surface emitting lasers," IEEE J. Quantum Electron. Vol. 27, No. 6, pp. 14021409, 1991.

[14] M.R. Park, O.K. Kwon, W.S. Han, K.H. Lee, S.J. Park, and B.S. Yoo, "All-epitaxial InAlGaAs-InP VCSELs in the 1.3-1.6-/spl $\mathrm{mu} / \mathrm{m}$ wavelength range for CWDM band applications," IEEE Photon. Technol. Letters. Vol. 18, No. 16, pp. 1717-1719, 2006.

[15] M. Muller, W. Hofmann, T. Grundl, M. Horn, P. Wolf, R. D. Nagel, E. Ronneberg, G.Bohm, D. Bimberg, and M. C. Amann, "1550-nm High-Speed Short-Cavity VCSELs," IEEE J. Selec. Topics in Quantum Electron. Vol. 17, No. 5, pp. 1158-1166, 2011.

[16] V. Jayaraman, J. Jiang, H. Li, P. Heim, G. Cole, B. Potsaid, J. G. Fujimoto, and A.
Cable,"OCT Imaging up to $760 \mathrm{Khz}$ Axial Scan Rate Using Single-Mode 1310nm MEMs-TunableVCSELs with \& gt;100nm Tuning Range," in CLEO, Baltimore, Maryland, USA, 2011.

[17] W. Hofmann, M. Muller, P. Wolf, A. Mutig, T. Grundl, G. Bohm, D. Bimberg, and M. C.Amann, "40 Gbit/s modulation of $1550 \mathrm{~nm}$ VCSEL," Electron. Lett. Vol. 47, No. 4, pp. 270-271, 2011.

[18] A. Karim, S. Bjorlin, J. Piprek, and J. Bowers, "Long-wavelength vertical-cavity lasers and amplifiers," IEEE J. Selec. Topics Quantum Electron, Vol. 6, No. 6, pp.1244-1253, 2000.

[19] G. B. Stringfellow and M.G. Craford, High Brightness Light Emitting Diodes San Diego: Academic, 1997.

[20]M. Hansen, J. Piprek, P.M. Pattison, J.S. Speck, S. Nakamura, and S.P DenBaars, "Higher efficiency InGaN laser diodes with an improved quantum well capping configuration," Appl. Phys. Lett. Vol. 81, pp.4275-4280, 2002.

[21]N. Tansu, Y. Chang, T. Takeuchi, D.P. Bour, S. W. Corzine, R. T. Tan, and L. J. Mawst, "Temperature Analysis and Characteristics of Highly Strained InGaAs-GaAsP-GaAs $(\lambda>$ $1.17 \mu \mathrm{m}) \quad$ Quantum-Well Lasers,'IEEE. Transac. Quantum Electron. Vol. 38, No. 6, pp. 640-651 2002.

[22]Z. Daneshkaftroudy and E. Rajaei, "Thermal simulation of InP - based $1.3 \mu \mathrm{m}$ vertical cavity surface emitting laser with AsSb- based DBRs," Opt. Commun. Vol. 284, pp. 330340,2011 .

[23]X. Li, Optoelectronic Devices: Design, Modeling and simulation, Cambridge University Press, 2009.

[24] M. Grupen and K. Hess, "Simulation of carrier transport and nonlinearities in quantum-well laser diodes," IEEE J. Quantum Electron. Vol. 34, No. 1, pp. 120-140, 1998. Carrier capture; Spectral hole burning; Helmholtz equation; Bethe thermionic emission theory.

[25]TcadSentaurs Device Optoelectronics http://www.synopsys.com/products/tcad/senta urus devopto ds.html.

[26]B. Witzigmann, A. Witzig, and W. Fichtner, "A full 3-dimensional quantum well laser simulation," Glasgow, UK, pp. 13 - 14, 2000. 
[27]"Tiber cad multi scale device simulator. "www.tibercad.org.

[28] Available online at www.crosslight.com.

[29]E.M. Azoff, "Energy Transport Numerical Simulation of Graded AlGaAs/GaAs Heterojunction Bipolar Transistors," IEEE Transact. Electron Devices, Vol. 36, No. 4, pp. 609-616, 1989.

[30]M.A. Alam, "Simulation of semiconductor quantum well lasers," IEEE Transact. on Electron Devices, Vol. 47, No. 10, pp. 19171925, 2000.

[31]Z. Danesh Kaftroudi, Esfandiar Rajaei, and A. Mazandarani, "Simulation of a Single-Mode Tunnel-Junction-Based Long Wavelength VCSEL," J. Russian Laser Research, Volume 35, Number 2,pp 124-137, 2014.

[32]J. Piprek, Semiconductor optoelectronic devices, Elsevier Science, San Diego,2003.

[33]T. Ouchi, "Thermal analysis of thin-film vertical-cavity surface-emitting lasers using finite element method," Jpn. J. Appl. Phys. Vol. 41,pp. 5181-5186.

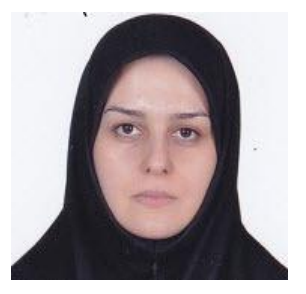

Zahra Danesh Kaftrouddiis an Associate Professor of Physics in the Department of Engineering sciences of Guilan University. She received her B.Sc. degree from Iran University of Science and Technology in 1998 and M.Sc. and Ph.D. degrees from Guilan University in 2003 and 2010, respectively.

She has academic skills in optoelectronic device simulation.

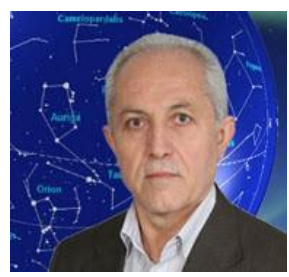

Esfandiar Rajaei received his B.Sc. degree in physics from the University of Tehran in 1976. He received his M.Sc. and $\mathrm{PhD}$ degrees from Montpellier University in 1981 and 1985, respectively. His research interest is mainly in the quantum dot laser simulation. 
THIS PAGE IS INTENTIONALLY LEFT BLANK. 